\title{
Status and prospects of the TAx4 experiment
}

\author{
Eiji Kido ${ }^{1, *}$ on behalf of the Telsecope Array Collaboration \\ ${ }^{1}$ Institute for Cosmic Ray Research, University of Tokyo, 5-1-5 Kashiwanoha, Chiba 277-8582, Japan
}

\begin{abstract}
The TAx 4 experiment is a project to observe highest energy cosmic rays by expanding the detection area of the TA experiment with newly constructed surface detectors (SDs) and fluorescence detectors (FDs). The construction of both SDs and FDs is ongoing. New SDs are arranged in a square grid with $2.08 \mathrm{~km}$ spacing at the north east and south east of the TA SD array. Field of view of new FDs overlaps the detection area of new SDs to observe SD FD hybrid events. New SDs are planning to be deployed early next year. The first light with new FDs was already observed on February 2018. Especially the hotspot and energy spectrum anisotropy are expected to be understood in more detail from the implications obtained by the TA experiment.
\end{abstract}

\section{Introduction}

Telescope Array (TA) is the largest cosmic-ray observatory in the Northern Hemisphere. The TA experiment has 507 surface detectors (SDs) on a square grid with $1.2 \mathrm{~km}$ spacing covering approximately $700 \mathrm{~km}^{2}$. The SDs are surrounded by three fluorescence detector (FD) stations (12, 12 and 14 telescopes). The duty cycle of the SD array is greater than $90 \%$ throughout 10 -year observation period, whereas the FD duty cycle is about $10 \%$ because the data is taken only on moonless clear nights. The locations of the detectors of TA are shown in Fig. 1. in Ref. [1]. The latitudes and the longitudes of the detector sites are around $39.30^{\circ} \mathrm{N}$ and $112.91^{\circ} \mathrm{W}$ in Utah in the USA.

The hotspot was observed in the arrival directions of 72 cosmic rays with energies above $57 \mathrm{EeV}$ [2]. The data used in the analysis was obtained by observing with TA SD for 5 years. The chance probability to exceed the obtained maximum significance $(5.1 \sigma)$ in an isotropy sky is estimated to be $3.4 \sigma$ in Ref. [2].

The SD array of the TAx 4 experiment was designed to study cosmic rays with energies especially above $57 \mathrm{EeV}$. The spacing of TAx 4 SD array is sparser than TA SD to obtain larger detection area. The spacing of the array is 2.08 $\mathrm{km}$. Cosmic rays with higher energies make larger number of SDs triggered and the sparse array does not lose the quality of event reconstructions very much at the higher energies. Reconstruction efficiency of the SD array is expected to be more than $95 \%$ above $57 \mathrm{EeV}$. 500 TAx4 SDs cover about 3 times larger area than 507 TA SDs and the combined coverage of TAx 4 SDs with TA SDs is about $3000 \mathrm{~km}^{2}$. Fig. 1. in Ref. [1] also shows the locations of TAx 4 detectors. 2 FD stations of the TAx 4 experiment were also designed. The field of view of FDs covers the detection area of SDs to observe SD FD hybrid events.

The implications of the energy spectrum anisotropy were recently observed by the TA experiment [3-5]. The

*e-mail: ekido@icrr.u-tokyo.ac.jp difference between the energy spectrum within 30 degrees from the supergalactic plane and the energy spectrum in the other part of the sky region was implicated in Ref. [3]. The statistical significance of the difference was estimated to be $3.2 \sigma$. The difference between the energy spectra above and below the declination $\delta=24.8^{\circ}$ was also implicated in Ref. [4]. The statistical significance of the difference of the break points of the energy spectra was estimated to be $3.5 \sigma$. The energy spectrum anisotropy above $E=10^{19.2} \mathrm{eV}$ was implicated in Ref. [5]. The post-trial significance of the anisotropy was estimated to be $3.74 \sigma$. These implications were obtained using TA SD data which was obtained from the observation for 5-7 years, and the implications are expected to be confirmed by the TAx 4 experiment.

Construction of the TAx4 detectors is now ongoing. The status of the construction of detectors is shown in the section 2. Prospects of the observation are shown in the section 3.

\section{Construction}

The status of the construction of SDs and FDs is shown in the section 2.1 and the section 2.2 , respectively.

\subsection{Construction of SDs}

The detailed structure of SDs is shown in Fig. 2 in Ref. [1]. The assembly of scinitillator boxes of SDs was started in 2015. The scintillator boxes were assembled in Japan every year and transported to the USA. The assembly in South Korea was also started in 2018. The quality of scintillator boxes is almost same as shown in the section 3 in Ref. [1]. SDs were assembled with the transported scintillator boxes were in Delta, Utah. Fig. 1 shows assembled SDs in Delta, Utah. 


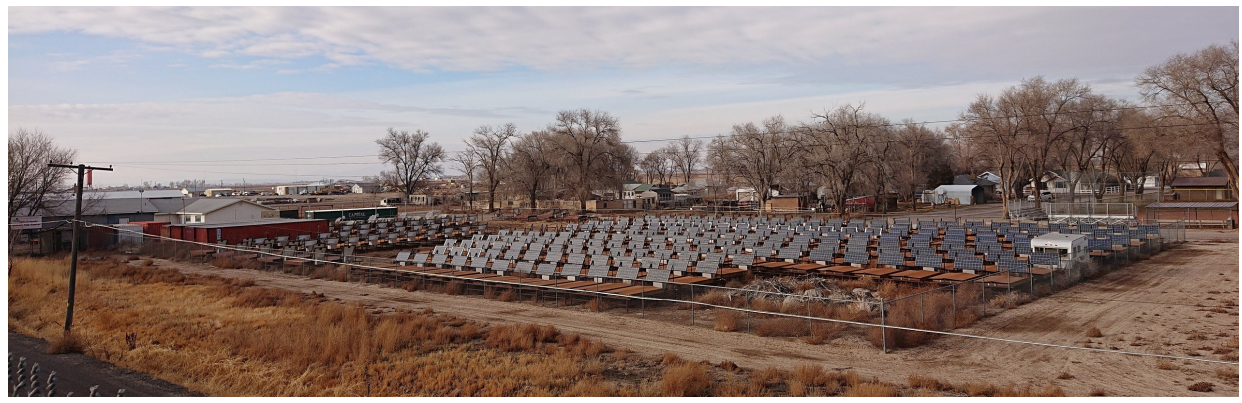

Figure 1. Picture of assembled SDs. These SDs will be deployed in the observation sites.

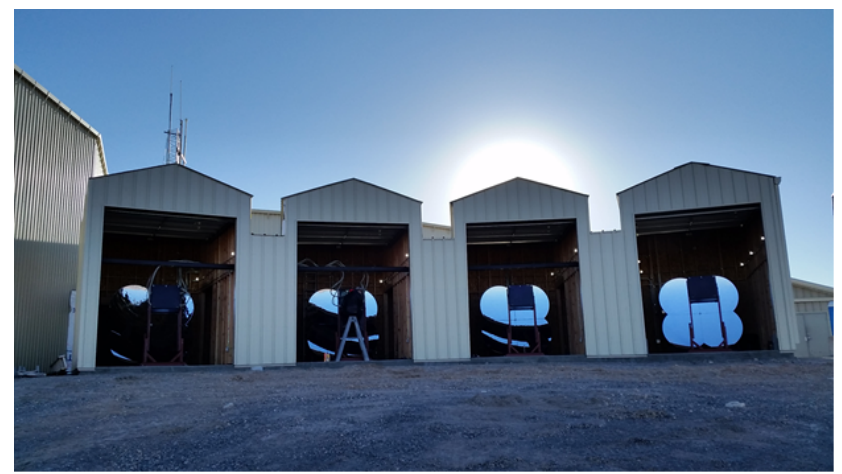

Figure 2. Picture of assembled 4 telescopes in the FD station at the north site of the TAx4 experiment.

\subsection{Construction of FDs}

2 FD stations are being constructed using transported telescopes of the HiRes experiment. North FD station was constructed, and the first light was observed on Feb. 16, 2018. Fig. 2 shows constructed north FD station. 4 and 8 telescopes are instrumented in the north and south FD station, respectively. Construction of the south FD station is ongoing.

\section{Prospects}

More than half of 500 SDs will be deployed in the observation sites early 2019 . The observation of SDs will be started after that. North FD station was already constructed, and the south FD station will be constructed soon.

The hotspot and energy spectrum anisotropy will be studied with 4 times larger statistics than TA SDs if the full operation is started. The full operation of the TAx4 experiment will provide about 3 times larger number of SD FD hybrid events than the TA experiment at the highest energies. Mass composition of cosmic rays will be studied in detail with the hybrid events.

\section{Acknowledgements}

The Telescope Array experiment is supported by the Japan Society for the Promotion of Science(JSPS) through Grants-in-Aid for Priority Area 431, for Specially Promoted Research JP21000002, for Scientific Research (S) JP19104006, for Specially Promoted Research JP15H05693, for Scientific Research (S) JP15H05741, for Science Research (A) JP18H03705 and for Young Scientists (A) JPH26707011; by the joint research program of the Institute for Cosmic Ray Research (ICRR), The University of Tokyo; by the U.S. National Science Foundation awards PHY-0601915, PHY-1404495, PHY-1404502, and PHY-1607727; by the National Research Foundation of Korea (2016R1A2B4014967, 2016R1A5A1013277, 2017K1A4A3015188, 2017R1A2A1A05071429); by the Russian Academy of Sciences, RFBR grant 16-02-00962a (INR), IISN project No. 4.4502.13, and Belgian Science Policy under IUAP VII/37 (ULB). The foundations of Dr. Ezekiel R. and Edna Wattis Dumke, Willard L. Eccles, and George S. and Dolores Doré Eccles all helped with generous donations. The State of Utah supported the project through its Economic Development Board, and the University of Utah through the Office of the Vice President for Research. The experimental site became available through the cooperation of the Utah School and Institutional Trust Lands Administration (SITLA), U.S. Bureau of Land Management (BLM), and the U.S. Air Force. We appreciate the assistance of the State of Utah and Fillmore offices of the BLM in crafting the Plan of Development for the site. Patrick Shea assisted the collaboration with 
valuable advice on a variety of topics. The people and the officials of Millard County, Utah have been a source of steadfast and warm support for our work which we greatly appreciate. We are indebted to the Millard County Road Department for their efforts to maintain and clear the roads which get us to our sites. We gratefully acknowledge the contribution from the technical staffs of our home institutions. An allocation of computer time from the Center for High Performance Computing at the University of Utah is gratefully acknowledged.

\section{References}

[1] E. Kido for the Telescope Array Collaboration, JPS Conf. Proc. 19, 011025 (2018)

[2] R. Abbasi et al., ApJ. 790, L21 (2014)

[3] R. Abbasi et al., arXiv: 1707.04967 [astro-ph.HE] (2017)

[4] R. Abbasi et al., arXiv: 1801.07820 [astro-ph.HE] (2018)

[5] R. Abbasi et al., ApJ. 862, 91 (2018) 